\title{
Gamma-ray bursts from tidally spun-up Wolf-Rayet stars?
}

\author{
R. G. Detmers ${ }^{1,2}$, N. Langer ${ }^{1}$, Ph. Podsiadlowski ${ }^{3}$, and R. G. Izzard ${ }^{1}$ \\ 1 Astronomical Institute, University of Utrecht, Postbus 80000, 3508 TA Utrecht, The Netherlands \\ 2 SRON National Institute for Space Research, Sorbonnelaan 2, 3584 CA Utrecht, The Netherlands \\ e-mail: r.g.detmers@sron.nl \\ 3 Department of Astrophysics, University of Oxford, Keble Road, Oxford OX1 3RH, UK
}

Received 9 January 2008 / Accepted 20 March 2008

\section{ABSTRACT}

\begin{abstract}
Context. The collapsar model requires rapidly rotating Wolf-Rayet stars as progenitors of long gamma-ray bursts. However, Galactic Wolf-Rayet stars rapidly lose angular momentum due to their intense stellar winds.

Aims. We investigate whether the tidal interaction of a Wolf-Rayet star with a compact object in a binary system can spin up the Wolf-Rayet star enough to produce a collapsar.

Methods. We compute the evolution of close Wolf-Rayet binaries, including tidal angular momentum exchange, differential rotation of the Wolf-Rayet star, internal magnetic fields, stellar wind mass loss, and mass transfer. The Wolf-Rayet companion is approximated as a point mass. We then employ a population synthesis code to infer the occurrence rates of the various relevant binary evolution channels.

Results. We find that the simple scenario - i.e., the Wolf-Rayet star being tidally spun up and producing a collapsar - does not occur at solar metallicity and may only occur with low probability at low metallicity. It is limited by the widening of the binary orbit induced by the strong Wolf-Rayet wind or by the radius evolution of the Wolf-Rayet star that most often leads to a binary merger. The tidal effects enhance the merger rate of Wolf-Rayet stars with black holes such that it becomes comparable to the occurrence rate of long gamma-ray bursts.
\end{abstract}

Key words. stars: binaries: close - stars: evolution - stars: Wolf-Rayet - gamma rays: bursts

\section{Introduction}

Although our understanding of long gamma-ray bursts (GRBs) has increased significantly since they were first discovered, it is still not clear what their progenitors exactly are. From studies of host galaxies it emerged that they occur in or near starforming regions and that several are associated with energetic type Ic supernova (Hjorth et al. 2003). These supernovae are thought to stem from to the explosion of a massive Wolf-Rayet (WR) star, although the signatures of a WR type progenitor have been unambiguously found in the afterglow of only one GRB (van Marle et al. 2005).

The most widely used model for the formation of GRBs is the collapsar model (Woosley 1993). In this model the core of a massive, fast rotating star collapses into a black hole. An accretion disk is formed around the black hole if the core has enough specific angular momentum, i.e. $j \gtrsim 3 \times 10^{16} \mathrm{~cm}^{2} \mathrm{~s}^{-1}$ (MacFadyen \& Woosley 1999). The remainder of the core is accreted onto the black hole and a highly relativistic collimated outflow is produced which releases a large amount of energy $\left(10^{51} \mathrm{erg}\right)$. If the star has no hydrogen envelope, the light crossing time is less or comparable to the duration of the accretion. In this case a GRB can be formed along with a type Ib/c supernova. Thus, rapidly rotating WR stars are required to produce a collapsar.

Earlier work on GRB progenitors (Petrovic et al. 2005; Hirschi et al. 2006) has shown that stellar models without a magnetic field can have enough specific angular momentum in their cores to produce a GRB within the collapsar model. The single star models in which magnetic fields according to Spruit (2002) were included had too little specific angular momentum in their cores due to the increased core-envelope coupling, which leads to a spin-down of the core (Petrovic et al. 2005; Heger et al. 2005). The reason why magnetic fields are considered to be important are the low observed rotation rates of young neutron stars and white dwarfs. In order to reproduce those low rates, magnetic torque need to be included (Heger et al. 2005; Suijs et al. 2008). Petrovic et al. (2005) also considered binary models in which the secondary star was spun up to close to critical rotation due to mass transfer from the primary. In their model, initially the core of the companion was spun up due to magnetic coreenvelope coupling, but the same mechanism decreased the core angular momentum by almost a factor of 100 once the star had reached core helium burning. Therefore, either one has to consider stars with a low metallicity which have a lower mass loss rate, or GRBs at solar metallicity require a more exotic binary channel. The first option has been considered recently by Yoon \& Langer (2005) and Yoon et al. (2006) and the second option is discussed here.

We investigate here whether tides in a close binary system can spin up a WR star enough so that it can form a collapsar. The magnetic fields inside the star enforce close to rigid rotation during core helium burning, so that angular momentum added to the envelope due to the tidal interaction is expected to be transported to the core of the WR star. In order to have a sufficiently strong tidal interaction for spin-up to occur, the orbital period needs to be smaller than about $24 \mathrm{~h}$. This restricts potential companions to compact stars, helium stars, or low mass main-sequence stars. While the number of double-WR star systems is too small to be significant (Vrancken et al. 1991), van den Heuvel \& Yoon (2007) showed that for main sequence companions, the obtained 
spin-up is insufficient to produce a GRB. Therefore, we focus on compact companions in the following sections.

Currently, only one compact WR binary is known in our Galaxy, namely Cyg X-3. The system has a period of $4.8 \mathrm{~h}$ and a period derivative in the range of $1-2 \times 10^{-6} \mathrm{yr}^{-1}$ (Lommen et al. 2005). Cyg X-3 has long been known as an X-ray binary. Van Kerkwijk et al. (1996) found that the primary is a WR-star, probably of the WN spectral type. The exact mass of the WR star is unknown, but Stark \& Saia (2003) have placed an upper limit on the mass of $7.3 M_{\odot}$ using observations taken with Chandra. Although the nature of the companion is still not clear, Van Kerkwijk et al. (1996) assume it is a neutron star of $1.4 M_{\odot}$, while Stark \& Saia (2003) place an upper limit of 3.6 $M_{\odot}$ on the compact object mass.

We assume a mass of $10 M_{\odot}$ for the WR star and $1.4 M_{\odot}$ for the compact object in our Cyg X-3 case study. The mass loss rate is highly uncertain, and estimated by various authors to be between $0.5 \times 10^{-6} M_{\odot} \mathrm{yr}^{-1}-2.9 \times 10^{-4} M_{\odot} \mathrm{yr}^{-1}$ (Lommen et al. 2005).

Systems similar to Cyg X-3, and those considered further on in this paper, can only be formed through common-envelope evolution. We use Cyg X-3 as a case-study for the tidal spin-up process to determine the effect of this process and to see whether it is capable of spinning up the WR star enough to meet the collapsar criterium. The remainder of this paper is organised as follows. In Sect. 2 we explain our physical assumptions as well as our numerical methods. Our binary evolution models are decribed in Sect. 3, while Sect. 4 contains our population synthesis results. Finally, our conclusions are discussed in Sect. 5.

\section{Methods and physical assumptions}

Our stellar models are calculated with a hydrodynamic stellar evolution code (Petrovic et al. 2005). Magnetic fields and the transport of angular momentum due to magnetic torque are included (Spruit 2002), as well as the effects of the centrifugal force on the stellar structure, chemical mixing and transport of angular momentum due to rotationally induced hydrodynamic instabilities, and enhanced mass loss due to close-to-critical rotation. The stellar wind mass loss of the WR star is calculated according to Hamann et al. (1995, labelled WR0 from now on) for WR stars with $\log L / L_{\odot}>4.5$ :

$\log \left(\dot{M}_{\mathrm{WR}} / M_{\odot} \mathrm{yr}^{-1}\right)=\epsilon \times\left(-11.95+1.5 \log L / L_{\odot}\right)$,

and Hamann et al. (1982) for helium stars with $\log L / L_{\odot}<4.5$ :

$\log \left(\dot{M}_{\mathrm{wR}} / M_{\odot} \mathrm{yr}^{-1}\right)=\epsilon \times\left(-35.8+6.8 \log L / L_{\odot}\right)$,

with $\dot{M}_{\mathrm{WR}}$ the mass loss rate of the Wolf-Rayet star and $\epsilon$ is a multiplication factor used to modify the mass loss rate.

When a star appoaches critical rotation, the mass loss of the star will be enhanced such that over-critical rotation is prevented. To achieve this, we follow the prescription of Langer (1997):

$\dot{M}=\dot{M}\left(V_{\text {rot }}=0\right)\left(\frac{1}{1-V_{\text {rot }} / V_{\text {crit }}}\right)^{0.43}$,

where

$V_{\text {crit }}=\sqrt{\frac{G M}{R}(1-\Gamma)}$.

Here $V_{\text {rot }}$ is the rotational velocity of the star, $\dot{M}$ is the mass loss rate of the star, $\Gamma=\kappa L /(4 \pi c G M)$ is the Eddington factor, and $\kappa$ is the opacity coefficient. We apply three different rates of mass loss, both based on Eqs. (1) and (2), using $\epsilon=0.1, \epsilon=1 / 3$, and $\epsilon=1$. We call these mass loss rates WR1, WR2, and WR3, respectively. The WR2 rate roughly agrees with the mass loss rate for Galactic WR stars (Nugis \& Lamers 2000). We chose the first rate to study the effect of a lower mass loss rate on the evolution of the system. This essentially captures the situation of a lower initial metallicity, since the mass loss rate of a WR star is dependant on its metallicity (Vink \& de Koter 2005), while all other properties depend very little on it.

For a given WR mass loss rate, one can define an angular momentum loss timescale, i.e. the time it takes for the star to lose most of its angular momentum to the stellar wind,

$\tau_{\text {ang }}=\frac{J_{\mathrm{WR}}}{\dot{J}_{\mathrm{WR}}}=\alpha \frac{M_{\mathrm{WR}}}{\dot{M}_{\mathrm{WR}}}$

where $M_{\mathrm{WR}}$ is the mass of the Wolf-Rayet star, $J_{\mathrm{WR}}$ is the total spin angular momentum of the star and $\alpha \simeq 0.1$ for efficient internal angular-momentum transport, since angular momentum loss from the wind is about 10 times faster than the mass loss (Packet 1981; Langer 1998).

There are two different mechanisms to synchronize a star with the orbit, the equilibrium tide and the dynamical tide. Although both mechanisms create a tidal bulge which causes the binary to become synchronized, they do so on different timescales. The time it takes for a star to synchronize with the orbit if it has a convective envelope is defined as (Zahn 1977):

$\tau_{\text {sync }} \propto q^{-2}\left(\frac{d}{R}\right)^{6}$

where $q=M_{\mathrm{cc}} / M_{\mathrm{WR}}$ is the mass ratio of the binary, with $M_{\mathrm{WR}}$ the total mass of the WR star and $M_{\mathrm{cc}}$ the mass of the compact companion, $d$ the orbital separation and $R$ the radius of the star to be synchronized. We choose to use this ansatz for the equilibrium tide for determining $\tau_{\text {sync }}$, although it was originally designed for convective stars, for two reasons. First, in the radiative envelopes of rapidly rotating stars, as considered here, rotation is supposed to produce a high level of turbulence, implying that turbulent viscosity dominates over the radiative viscosity. Second, Toledano et al. (2007), applying a kinematic model of tidal interaction to early type stars, found that the tidal time scale in these models indeed scales as $(d / R)^{6}$.

The angular momentum exchange between the orbit and the WR spin is computed in the following way (Wellstein 2001). If $\Delta J$ is the amount of spin angular momentum that is added to the star in the considered time step $\Delta t$ due to tides then,

$\Delta J=\left(J_{\mathrm{WR}}-J_{\text {sync }}\right)\left(1-\mathrm{e}^{-\Delta t / \tau_{\text {sync }}}\right)$,

where $J_{\text {sync }}$ is the spin angular momentum the star would have when the system is synchronized.

Angular momentum $\Delta J$ is added to the outer layers at every time step. Angular momentum transport processes (i.e. magnetic torque) redistribute the angular momentum over the whole star. Magnetic torque is strong enough to keep the star close to rigid rotation during core helium burning. In this way the whole of the WR star is spun up due to the tidal interaction.

To determine the outcome of the common-envelope evolution we calculate the final orbital separation and compare that to the $\mathrm{CO}$-core radius and the Roche-lobe radius of the $\mathrm{CO}$-core. We do this using the energy equation for common envelope evolution (Webbink 1984; de Kool 1990):

$\frac{a_{\mathrm{f}}}{a_{\mathrm{i}}}=\frac{M_{\mathrm{core}} M_{\mathrm{cc}}}{M_{\mathrm{WR}}} \frac{1}{M_{\mathrm{cc}}+2 M_{\mathrm{env}} a_{\mathrm{i}} /\left(\eta_{\mathrm{CE}} \lambda R_{\mathrm{L}}\right)}$, 
Table 1. Cyg X-3 models.

\begin{tabular}{ccccc}
\hline \hline$M_{\mathrm{WR}}\left[M_{\odot}\right]$ & $M_{\mathrm{CC}}\left[M_{\odot}\right]$ & $P_{i}[\mathrm{~h}]$ & $\epsilon$ & Model \\
\hline 10 & 1.4 & 4.8 & 1 & $\mathrm{WRa}$ \\
10 & 1.4 & 4.8 & 0.1 & $\mathrm{WRb}$ \\
10 & 1.4 & 4.8 & 0.01 & $\mathrm{WRc}$ \\
\hline
\end{tabular}

where $a_{\mathrm{f}}$ is the final orbital separation after the CE phase and $a_{\mathrm{i}}$ the orbital separation at the moment when Roche Lobe overflow (RLOF) starts. $M_{\text {core }}$ is the mass of the CO-core of the WR star and $M_{\text {env }}$ the mass contained in the envelope of the WR star. The efficiency parameter $\eta_{\mathrm{CE}}$ is set to 1 in our calculations and $\lambda$ is determined by the binding energy of the envelope, which is extracted from our models.

\section{Binary evolution models}

We first compute tailored models for Cyg X-3 to determine the role of tidal interaction in this system. We assume a compact object mass of $1.4 M_{\odot}$ (i.e. take a neutron star), chose a WR-star mass of $10 M_{\odot}$ and set the initial orbital period to $4.8 \mathrm{~h}$. The mass loss rates for our Cyg X-3 models are based on the Hamann et al. (1995) rate, but with $\epsilon=1$ (model WRa), $\epsilon=0.1$ (WRb) and $\epsilon=0.01$ (WRc, see Table 1). Their evolution is described in the next subsection.

\subsection{Cyg X-3 models: a case study}

The three Cyg X-3 models which we have calculated show the effects of different mass loss rates on the tidal interaction and evolution of the system. The model sequences end either at carbon depletion in the core or when the primary fills its Roche-lobe. In all three cases the tidal interaction transfers angular momentum from the orbit to the WR star, but the WR star is only spun up if the mass loss rate is not too high, i.e. if $\tau_{\text {sync }}<\tau_{\text {ang. }}$. This is the case for models WRb and WRc, but for model WRa the mass loss rate is too high and thus $\tau_{\text {ang }}$ too small.

Figure 1 shows the mass loss rates for models WRa, WRb and WRc as function of time. The WRa mass loss rate decreases throughout the evolution and only increases sharply at $9.5 \times 10^{5} \mathrm{yr}$ at core helium exhaustion, because the luminosity increases greatly when the WR star starts its He-shell burning. The WRb and WRc mass loss rates are initially so low that the tides spin up the stars to critical rotation, which occurs here well before the WR stars fill their Roche lobes since their luminosities are near their Eddington luminosities (cf. Eq. (4)). The high luminosity of the WR star has the consequence that the star can approach critical rotation for $\omega_{\mathrm{WR}}<\omega_{\mathrm{orb}}$. Once at critical rotation, the mass loss rate is determined by the mechanical constraint to avoid over-critical rotation (cf. Langer 1998). While this is a numerically (and perhaps physically) unstable situation (cf. Fig. 3 in Langer 1998), the corresponding large oscillations of the mass loss rate have no consequences for the evolution of the system, as long as the long-term average of the mass loss rate is still well defined, as is the case here (cf. Sect. 3.1.2). As the orbital period decreases, the time-averaged mass loss rates of systems WRb and WRc increase in the course of evolution.

The time evolution of the orbital separation of these three sequences is shown in Fig. 2. The orbital separation of models WRb and WRc decreases, while it increases for model WRa. The evolution of model WRa is dominated by its mass loss. The two kinks which occur at $3 \times 10^{5}$ and $9.6 \times 10^{5} \mathrm{yr}$ can be traced

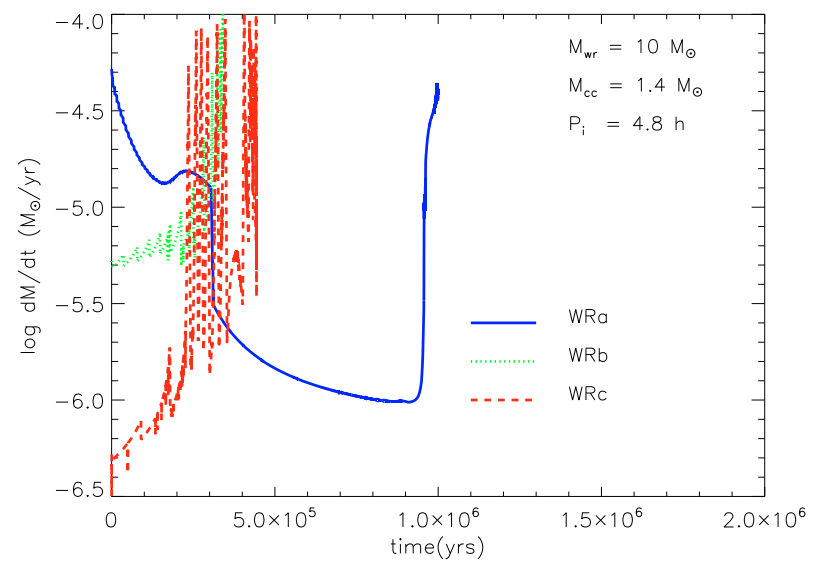

Fig. 1. mass loss rate as a function of time for the three Cyg X-3 models: WRa (blue line, solid) with $\epsilon=1$, WRb (green line, dotted) with $\epsilon=0.1$ and WRc (red line,striped) with $\epsilon=0.01$ (cf. Table 1).

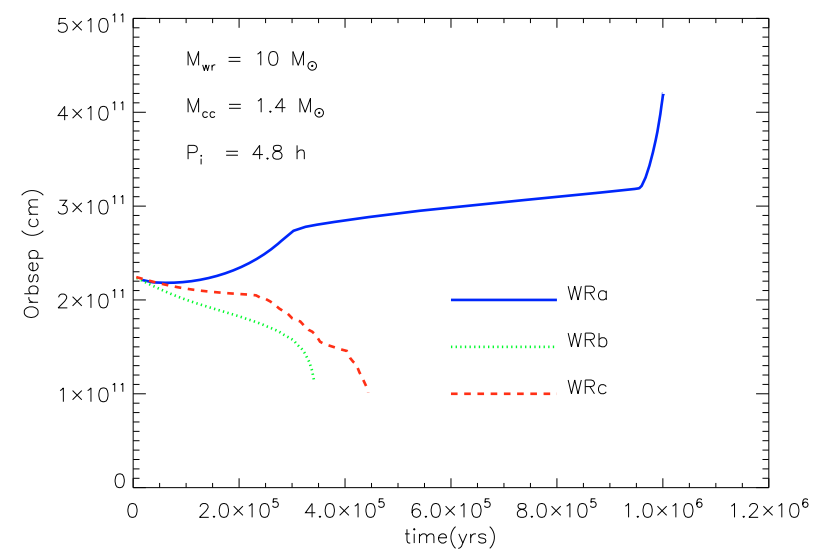

Fig. 2. Orbital separation as a function of time for our 3 different Cyg X-3 models. Plotted are model WRa (solid line), model WRb (dotted line) and the model WRc (dashed line).

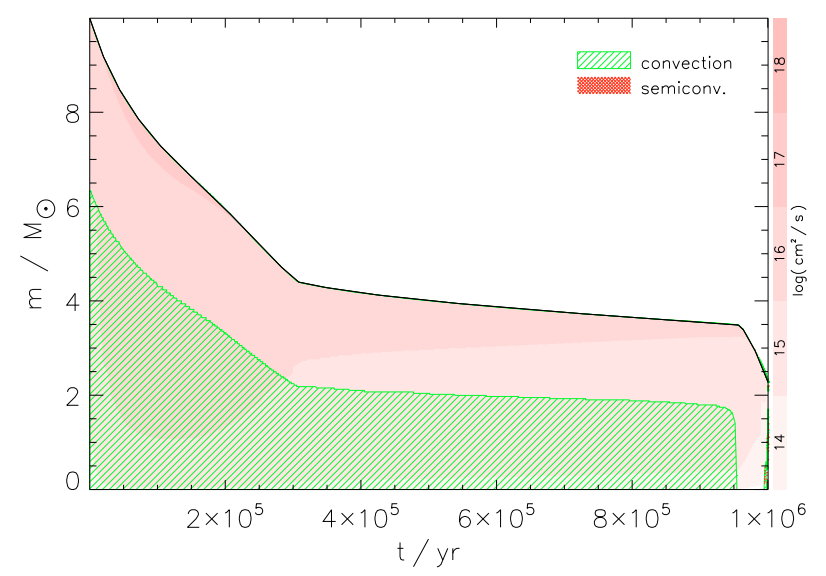

Fig. 3. Kippenhahn diagram of the specific angular momentum for the WR star in model WRa. The hatched and crossed areas indicate convection and semi-convection respectively. The red color indicates the amount of specific angular momentum, where a darker red indicates larger specific angular momentum.

back to the changes in the mass loss rate at those times (see Fig. 1). In models WRb and WRc the initial mass loss rate is low, so that the tidal interaction spins the star up and angular momentum is thus transferred from the orbit to the WR star. That 


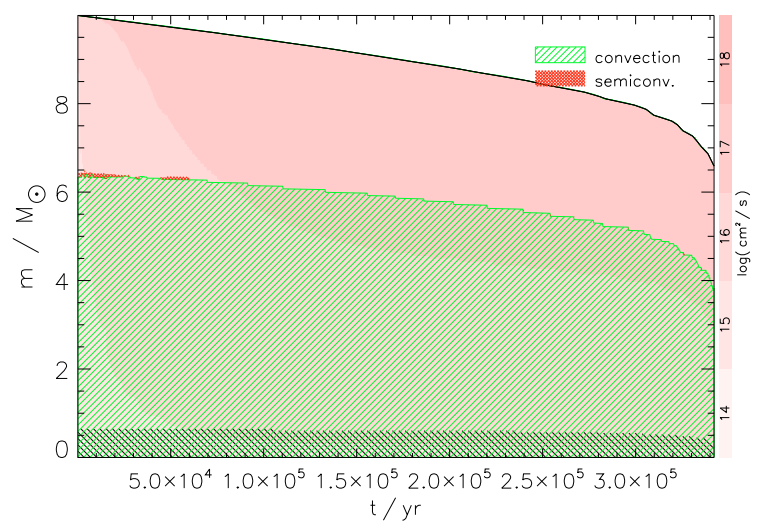

Fig. 4. As Fig. 3 except this is for model WRb. The transport of angular momentum from the envelope to the core can clearly be seen after $80000 \mathrm{yr}$.

means that the orbit shrinks and this process continues until the WR star starts to fill its Roche-lobe.

\subsubsection{Sequence WRa}

Model WRa reaches carbon depletion in the core, although the mass of the WR star is only $2.2 M_{\odot}$ at that time. The specific angular momentum of model WRa does not change significantly during the core helium burning phase, implying that the wind induced loss of spin angular momentum is compensated by tidally induced angular momentum gain. At the end of core carbon burning, the specific angular momentum drops to very low values in the core. This is due to the overwhelming effect of the increased mass loss, which brings the WR star out of tidal locking.

The internal specific angular momentum distribution during the evolution of the system can be seen in Fig. 3. The angular momentum in the core stays roughly constant throughout the evolution, except for an initial increase during the first $40000 \mathrm{yr}$ due to tidal spin-up, and a large decrease after helium shell burning has started at $9.5 \times 10^{5} \mathrm{yr}$. When $50 \%$ helium is left in the core, the star has already lost more than half of its mass due to its strong stellar wind. The WR star will not form a black hole because the $\mathrm{CO}$ core mass is too low (below $2 M_{\odot}$ ), but it will most likely form a neutron star.

\subsubsection{Sequences WRb and WRc}

Model WRb has an entirely different evolution. The star is spun up due to tidal interaction and as a consequence its specific angular momentum increases. At the end of the calculation, the specific angular momentum at $3 M_{\odot}$ is $10^{17} \mathrm{~cm}^{-2} \mathrm{~s}^{-1}$. Figure 4 shows the internal specific angular momentum distribution during the evolution. Because the orbital separation decreases with time, the star remains tidally locked and is spun up throughout the whole evolution. Figure 4 also shows that despite the oscillations of the mass loss rate as function of time (Fig. 1), the time average mass loss rate is well defined, and in fact dictated by the need to avoid faster than critical rotation at the stellar surface. Even though the star loses about 3.5 $M_{\odot}$ the star is still spinning rapidly at the moment RLOF occurs. The outcome of the RLOF phase will most likely be a merger, since the mass transfer process is unstable because of the large mass ratio.

The evolution of model WRc is similar to that of model $\mathrm{WRb}$, except that the orbital shrinking is less severe in this model. Although this may seem strange at first because the mass

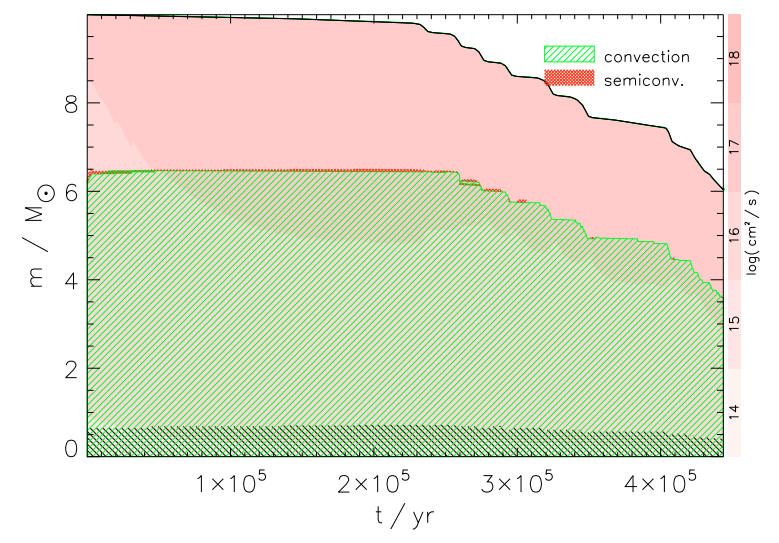

Fig. 5. As Fig. 3 except this is for model WRc.

loss rate is lower and thus the tidal interaction should be more effective in spinning up the star, the stronger mass loss in model $\mathrm{WRb}$ actually helps to decrease the orbital separation. This is because the system is tidally locked in both models such that any angular-momentum loss from the star comes from the orbit instead. This effect can be compared to magnetic braking in lowmass stars with a convective envelope. Since the mass loss rate is higher in model WRb, the angular momentum loss is also larger and the orbit shrinks faster.

Figure 5 shows the internal specific angular momentum as function of time. Again, the increase of specific angular momentum can be seen by the increase of the dark red area in the star. Interestingly, the final mass of the WR star is smaller in model WRc than in model WRb. This is due to several effects. First, although the initial mass loss rate is different for models WRb and WRc, the effect of the spin-up is that the WR star approaches critical rotation. The mass loss rate increases according to Eq. (3). The initial value of the mass loss rate does not matter once the star approaches critical velocity, which is why models WRb and WRc have an almost equal average mass loss rate. Secondly, the orbital decay of model WRc is less severe and so the point at which the WR star fills its Roche-lobe is reached at a later time, so the mass of the WR star is smaller when RLOF starts. The likely outcome of this RLOF is again a merger due to unstable mass transfer, as is the case for model WRb.

\subsubsection{Summary}

In the three Cyg X-3 models discussed above, either the spinup works and the orbit shrinks (models WRb and WRc) or the mass loss rate is too high and the orbit widens (model WRa). The WR star is initially spun up by the tidal interaction in model WRa, but after 50000 yr the star starts to lose angular momentum due to the strong stellar wind. Models WRb and WRc also spin-up initially, but retain their angular momentum throughout their evolution. The angular momentum of the orbit always decreases, due to the mass loss and the tidal interaction. All three systems synchronize their orbits very quickly and remain synchronized throughout.

From these three models it is clear that there is a threshold value for the mass loss rate which determines the outcome of the system. Either the mass loss rate is too high so the WR star spins down and the orbit widens, or the rate is low enough so that the WR star is spun up. The mass loss rate is determined not by the properties of the WR star, but rather by the need of sufficient angular-momentum loss to not exceed the $\Omega$-limit. Considering the limiting case of the mass loss rate approaching zero, it is 
clear that the tidal interaction is strong enough to spin up the star and thus the orbit would shrink. The star would then eventually approach critical rotation, and the mass loss is enhanced to a value which is high enough to keep the star from reaching critical rotation. So the spin-up works as shown in the WRb and WRc systems, but both systems merge in the end.

\subsection{Parameter study}

It is clear that a study of only Cyg X-3 is insufficient to investigate the whole parameter space. We therefore set up a grid of binary models with a mass of the WR star between 6 and $18 M_{\odot}$. Our models all have solar metallicity, and we take a zero-age helium main sequence star (ZAHeMS) as our WR star. We use different masses for the compact object: $1.4 M_{\odot}, 3.0 M_{\odot}$ and $5.0 M_{\odot}$, i.e. assume either a neutron-star or a black-hole companion. While larger compact object masses are possible, systems with such are expected to contribute at most 10-20\% to the total number of helium stars plus compact object systems. The initial orbital separation is chosen such that the synchronization timescale $\tau_{\text {sync }}$ is about equal to the stellar wind induced angular momentum loss timescale $\tau_{\text {ang }}$. The other chosen initial separations are twice and thrice this equilibrium value. For the $5 M_{\odot}$ companion we chose an extra set of models, with an initial orbital separation of 0.5 times the equilibrium value.

As shown for the Cyg X-3 models above, if the spin-up works then the orbital shrinking will most likely lead to RLOF. To investigate whether we can have tidal interaction to spin-up the WR star without ensuring RLOF we expanded our calculations to several model grids. We use the two different mass loss rates labelled as WR1 and WR2 (see Sect. 2). Each of the plots in Fig. 6 shows the mass of the WR star and the initial period for each system. We computed the evolution of each system and gave each different outcome a separate symbol in the plot. We find 5 different outcomes for the binary system.

The first and easiest to understand is the case where the initial orbital period is large. The tidal interaction is weak in these systems and mass loss dominates the evolution. The binary widens and the WR star spins down. Depending on the mass of the WR star it will form either a black hole or a neutron star, but in neither case is it spinning fast enough to be considered a GRB progenitor.

The second and third type of evolution occur when the tidal interaction is not strong enough to spin-up the WR star, so mass loss still widens the orbit. However, the system remains compact enough for the WR star to fill its Roche-lobe during He-shell burning. Unstable mass transfer starts at that moment and the result is a common-envelope phase (the second one in the evolution of the binary). We have found two possible outcomes for the common-envelope phase, either a merger, or the binary survives the common-envelope phase and ends up as a $\mathrm{CO}$ core with a compact object in a very small orbit.

The fourth type is the case where the tidal interaction is strong enough to spin up the WR star. The orbits shrinks and the WR star fills its Roche-lobe during core He-burning. If the mass ratio is too far from unity, the mass transfer is unstable and the result is a merger of both stars. When the compact companion is a neutron star the result will be a Thorne-Żytkow like object (Thorne \& Żytkow 1975). In the case of a black hole companion, the WR star will be accreted onto the black hole and a GRB may occur (we will discuss this option in the next section).

In the last type of evolution, the tidal interaction is initially strong enough to spin up the WR star to close to critical rotation and the orbit shrinks. Due to the WR star approaching critical rotation, the mass loss rate increases, which reduces the radius of the WR star, resulting in a weaker tidal interaction. Eventually the system is no longer tidally locked. The orbit widens again and the star continues to lose a large amount of mass. The end result is the same as for the systems with a large initial period.

We do not make a full grid calculation for the WR2 mass loss rate models, as the main effect of a higher mass loss rate is a shift of the borderlines between the various types of evolution in the WR1 grids to lower initial periods. The WR2 models are similar to the WR1 models, but the mass loss is higher and thus the initial equilibrium period is smaller. This is because $\tau_{\text {ang }}$ is smaller, which means that $\tau_{\text {sync }}$ also has to be smaller in order to have an equilibrium situation. The only potential collapsar progenitors are those systems which survive the common-envelope phase as a $\mathrm{CO}$ star with a compact companion in a close orbit, if the $\mathrm{CO}$ star is spinning fast enough and is massive enough to form a black hole. The $\mathrm{CO}$ core mass of a $10 M_{\odot}$ WR star is $6.0 M_{\odot}$, which may be close to the borderline value for forming a black hole. We have one possible system in our grid for which this could be the case, namely the $10 M_{\odot}$ WR star with a $3.0 M_{\odot}$ companion and an inital period of $23.15 \mathrm{~h}$ (see Table A.1). Out of the 70 systems we calculated with the WR1 mass loss rate, 19 systems may allow GRB production. The most important conclusion drawn from these models is that there is no model in which only tidal spin-up works, whilst avoiding RLOF or a merger. So our initial idea of having only tidal spin-up is unlikely to happen at these mass loss rates and thus at solar metallicity. Tidal interaction is very important for triggering the RLOF or a merger, thus indirectly contributing to the formation of a possible GRB. If tidal interaction is weaker than we have assumed here, then the equilibrium period will shift to a smaller initial orbital period, the same effect as a higher mass loss rate. If the tidal interaction is stronger than we have assumed, then the initial orbital period will shift upwards, the same effect as a lower mass loss rate. We did not change the strength of the tidal interaction, but making the tidal interaction stronger could be one way of making tidal spin-up work at solar metallicity, although the increase would have to be significant because of the high mass loss rates at solar metallicity.

We have investigated the possibility of tidal spin-up in a close WR-compact object binary leading to a collapsar. Our results show that it is very hard to have tidal spin-up while also avoiding a RLOF or merger event. At the same time however the tidal interaction is the cause of the pre He-shell burning mergers. These mergers may or may not produce a GRB, which is unknown at the moment. So indirectly tidal interaction may lead to the formation of a GRB in these cases. Even if these sources would not produce a GRB, they may appear as a transient source on the sky. So our results may have significant observable implications, since big efforts are undertaken to investigate transient sources at various wavelengths.

At lower metallicity (and lower mass loss rates), the range in periods at which tidal interaction is strong enough to spin up the WR star is larger. This is because the "equilibrium period" shifts to a larger initial period, because $\tau_{\text {ang }}$ is larger and thus also $\tau_{\text {sync }}$ can be larger. There is a limit to which lowering the mass loss rate makes a difference, because if the WR star approaches critical rotation due to the spin-up, the mass loss also increases and is determined by the angular momentum loss needed to avoid exceeding the critical rotation rate. So the inital mass loss rate may only be important in the initial evolution of the system. One way in which there could be only tidal spin-up would be if the initial mass loss rate is low and the initial orbital period is large enough so that the WR star does not approach critical rotation 

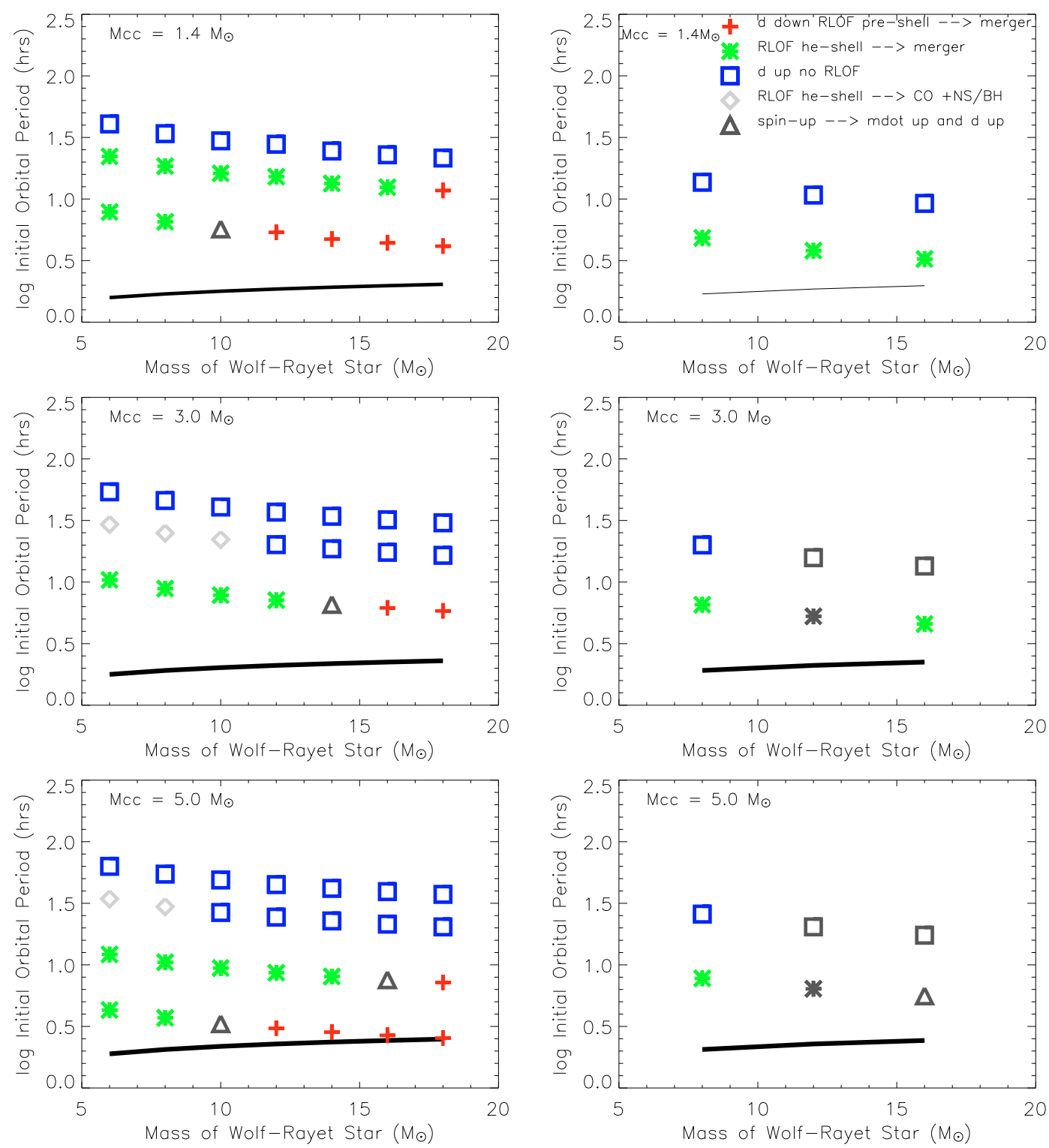

Fig. 6. The outcome of the evolutionary calculations for helium star-compact object binaries using the WR1 mass loss rate (left column) and the WR2 mass loss rate (right column). The companion mass is shown in the upper left corner of each plot. We take a $1.4 M_{\odot}$ companion (neutron star), a 3.0 $M_{\odot}$ companion (black hole) and a 5.0 $M_{\odot}$ companion (black hole). Evolution outcomes are: no RLOF and no spin-up (squares), spiral-in and RLOF during helium burning (crosses), RLOF during He-shell burning resulting in a merger (stars), RLOF during He-shell burning resulting in a CO-core + compact object in a close binary (diamonds), and spin-up then increased mass loss and orbital widening (triangles). The solid line indicates where the WR star would immediately fill its Roche-lobe, which gives the lower boundary for possible initial periods.

until the end of its evolution. In that way the WR star would have no time to spin down again and it will have enough angular momentum in its core to form a collapsar. So at lower mass loss rates, having only tidal spin-up is still a fine-tuning process, which means that it is unlikely that this scenario will be a major GRB production channel. Lowering the mass loss rate, i.e. assuming lower metallicity, will have the effect that tidal interaction leading to a RLOF or merger occurs more frequently, since the range of periods in which this occurs is wider than at solar metallicity.

\section{Population synthesis}

To estimate the birth rate of these kind of systems, we have performed a population synthesis study using the model of Hurley et al. (2002). A $100^{3}$ grid in $M_{1}, M_{2}$ and $d_{\text {init }}$ with grid limits
$13<M_{1} / M_{\odot}<55,6.5<M_{2} / M_{\odot}<40$ and $60<d_{\text {init }} / R_{\odot}<$ 1370 , which were chosen after searching a wide parameter space for progenitor systems, was searched for systems which match our GRB progenitors. We used the initial mass function of Kroupa, Tout, \& Gilmore (1993) for the primary, a flat distribution in $q=M_{1} / M_{2}$ between 0 and 1 and a separation distribution flat in $\log d_{\text {init }}$ between 3 and $10^{4} R_{\odot}$, a Maxwellian supernova kick velocity with dispersion $190 \mathrm{~km} \mathrm{~s}^{-1}$, solar metallicity $(Z=0.02)$, mass loss according to Hurley et al. (2002), circular orbits, common envelope parameters $\alpha=1.0$ and $\lambda=0.5$ or 0.05 , compact object (post-SN) masses according to Belczynski et al. (2002) and the star formation rate given in Hurley et al. (2002) of one binary with $M_{1}>0.8 M_{\odot}$ per year.

The formation rates of each of our potential GRB progenitors are given in Table 2 . We only consider systems that have a black hole as a companion. These systems can be grouped into three 
Table 2. Formation rates for each possible GRB progenitor type, for $\lambda=0.5$.

\begin{tabular}{cccc}
\hline \hline Scenario & Type & Fate & Birthrate $\left[\mathrm{yr}^{-1}\right]$ \\
\hline A & He-shell RLOF & CO-BH merger & $5.64 \times 10^{-6}$ \\
B & pre He-shell RLOF & He-BH merger & $3.83 \times 10^{-5}$ \\
C & CO + BH & collapsar? & $1.39 \times 10^{-7}$ \\
\hline
\end{tabular}

categories, each with a different evolutionary path. Group A consists of systems that avoid a RLOF phase during the core helium burning phase of the WR-star evolution, but experience RLOF and a merger during the consecutive expansion of the envelope when helium shell burning starts. Group B contains the systems which have a strong tidal interaction during core helium burning so that RLOF begins prior to the helium shell burning expansion of the star. These systems all end up as mergers. We also have one scenario, Group C, which we consider a possible collapsar progenitor scenario: The systems that survives the RLOF during helium shell burning and end up as $\mathrm{CO}$ star with a black hole companion. Here, the CO star is likely spun up by the tides and the remaining life time may be too short for mass loss or expansion of the WR star to be significant.

The systems in Group B have a significantly higher formation rate than systems in the the other two groups. A comparison of the formation rate of these systems to the average formation rate of GRBs per galaxy, which is $10^{-5} \mathrm{yr}^{-1}$ according to Cherepashchuk \& Postnov (2001), shows that these systems could account for a significant fraction of the GRBs or transient sources.

As with all population synthesis studies our results suffer from uncertainties in the input distributions and physics. Of particular note for the current work is the highly uncertain common-envelope evolution and associated free parameter combination $\alpha \lambda$. We chose those values to match previous studies, such as Hurley et al. (2002), but we vary $\lambda$ to show its considerable effect. A higher value for $\lambda$ leads to a less tightly bound common envelope and a higher chance of envelope ejection thus enhancing the chance to form close WR star plus compact object binaries. Reducing $\lambda$ to 0.05 reduces the rate by a factor of about 10. This can be understood since a lower $\lambda$ means a more tightly bound envelope, i.e. less systems are able to eject the envelope in the $\mathrm{CE}$ phase and merge. The compact object mass distribution (NS/BH) is also quite uncertain but we have no better prescription than that used here.

\section{Conclusions}

This paper shows that it is not a simple matter to spin up a WR star through tidal interaction and thereby produce a long gamma-ray burst. While the spin-up process itself may work, either the evolution of the binary orbit or of the radius of the WR star prevent the desired result, in almost all cases. Only at low metallicity, where the WR star winds may be weak and the orbit can thus be more stable, can this scenario work for a limited and rather insignificant fraction of the parameter space.

This negative result does not exclude something interesting happening to WR stars with a close companion: most systems with orbital periods below $\sim 20 \mathrm{~h}$ lead to a merger. In principle, the companion could be a compact object, a helium star, or a main sequence star. As the spin-up scenario fails, only the latter seems interesting in the context of gamma-ray burst formation (although see Fryer \& Heger 2005), in particular when the compact object is a black hole. While the merger of a helium star with a black hole or neutron star does not form a collapsar, it has been proposed that such events do produce long gammaray bursts (Fryer et al. 1999). However, detailed models of such mergers which can demonstrate their ability to produce a GRB are still missing. From the binary models presented above, we would expect two types of these events, i.e. a merger during or after core helium buring. Both may have rather long time scales compared to the average time scale of long gamma-ray bursts. It is also unclear whether these mergers can produce an explosive event resembling a Type Ib/c supernova. Even if these mergers do not produce a GRB, they may be observed as a transient source in the sky.

In summary, the main product of close WR binaries with compact companions is a helium star-compact object merger not a collapsing and rapidly rotating WR star. The occurance rate of these events may be compatible with that of long/soft gammaray bursts.

Acknowledgements. We are grateful to Arend-Jan Poelarends for excellent technical assistance, as well as Marten van Kerkwijk for discussion concerning Cyg X-3. RGI thanks NWO for his current fellowship in Utrecht.

\section{Appendix A: Grid models setup}

Table A.1 gives an overview of our grid of models using the WR1 mass loss rate, the models using the WR2 rate are shown in Table A.2.

Table A.1. Binary model properties for the WR1 mass loss rate.

\begin{tabular}{cccccc}
\hline \hline$M_{\mathrm{WR}}\left[M_{\odot}\right]$ & $M_{\mathrm{CC}}\left[M_{\odot}\right]$ & $P_{1}[\mathrm{~h}]$ & $P_{2}[\mathrm{~h}]$ & $P_{3}[\mathrm{~h}]$ & $P_{4}[\mathrm{~h}]$ \\
\hline 6 & 1.4 & 7.83 & 22.16 & 40.70 & - \\
8 & 1.4 & 6.53 & 18.47 & 33.94 & - \\
10 & 1.4 & 5,71 & 16.16 & 29.68 & - \\
12 & 1.4 & 5.14 & 14.55 & 26.73 & - \\
14 & 1.4 & 4.72 & 13.36 & 24.55 & - \\
16 & 1.4 & 4.40 & 12.45 & 22.86 & - \\
18 & 1.4 & 4.14 & 11.72 & 21.52 & - \\
\hline 6 & 3.0 & 10.40 & 29.41 & 53.99 & - \\
8 & 3.0 & 8.84 & 25.00 & 45.93 & - \\
10 & 3.0 & 7.83 & 23.15 & 40.69 & - \\
12 & 3.0 & 7.12 & 20.13 & 36.98 & - \\
14 & 3.0 & 6.58 & 18.61 & 34.20 & - \\
16 & 3.0 & 6.16 & 17.44 & 32.03 & - \\
18 & 3.0 & 5.82 & 16.48 & 30.28 & - \\
\hline 6 & 5.0 & 12.14 & 34.34 & 63.08 & 4.29 \\
8 & 5.0 & 10.49 & 29.69 & 54.55 & 3.71 \\
10 & 5.0 & 9.41 & 26.62 & 48.91 & 3.33 \\
12 & 5.0 & 8.63 & 24.41 & 44.85 & 3.05 \\
14 & 5.0 & 8.04 & 22.74 & 41.77 & 2.84 \\
16 & 5.0 & 7.57 & 21.41 & 39.34 & 2.68 \\
18 & 5.0 & 7.19 & 20.33 & 37.35 & 2.54 \\
\hline
\end{tabular}

Table A.2. Same as Table A.1, except the WR2 mass loss rate is used.

\begin{tabular}{ccccc}
\hline \hline$M_{\mathrm{WR}}\left[M_{\odot}\right]$ & $M_{\mathrm{CC}}\left[M_{\odot}\right]$ & $P_{1}[\mathrm{~h}]$ & $P_{2}[\mathrm{~h}]$ & $P_{3}[\mathrm{~h}]$ \\
\hline 8 & 1.4 & 4.83 & 13.67 & - \\
12 & 1.4 & 3.81 & 10.77 & - \\
16 & 1.4 & 3.26 & 9.21 & - \\
\hline 8 & 3.0 & 6.54 & 20.02 & - \\
12 & 3.0 & 5.27 & 15.76 & - \\
16 & 3.0 & 4.56 & 13.48 & - \\
\hline 8 & 5.0 & 7.77 & 25.84 & 2.75 \\
12 & 5.0 & 6.39 & 20.35 & 2.26 \\
16 & 5.0 & 5.60 & 17.41 & 1.98 \\
\hline
\end{tabular}




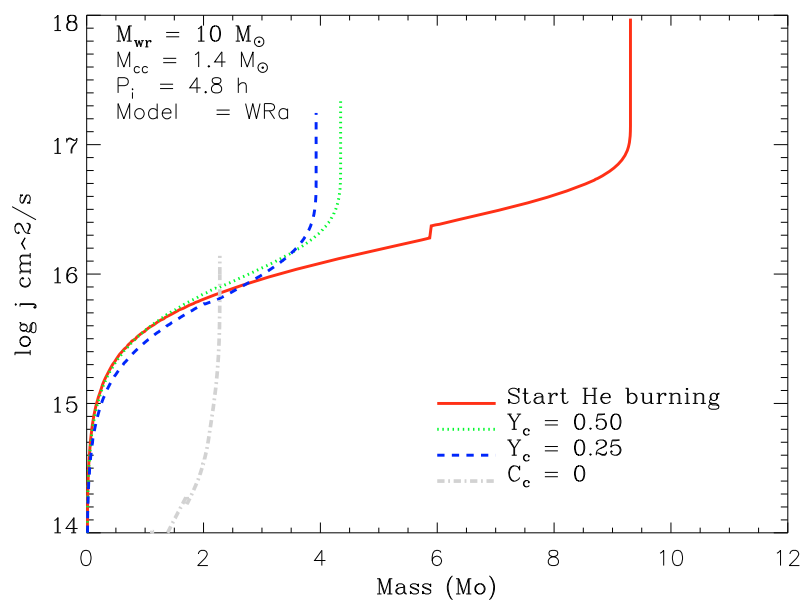

Fig. B.1. Specific angular momentum as a function of mass coordinate for our Cyg X-3 model WRa (1.4 $M_{\odot}$ companion). Profiles are for the start of core helium burning (solid line), $50 \%$ He-depletion in the core (dotted line), 25\% He-depletion in the core (dashed line) and the last model calculated when carbon is depleted in the core (dot-dashed line).

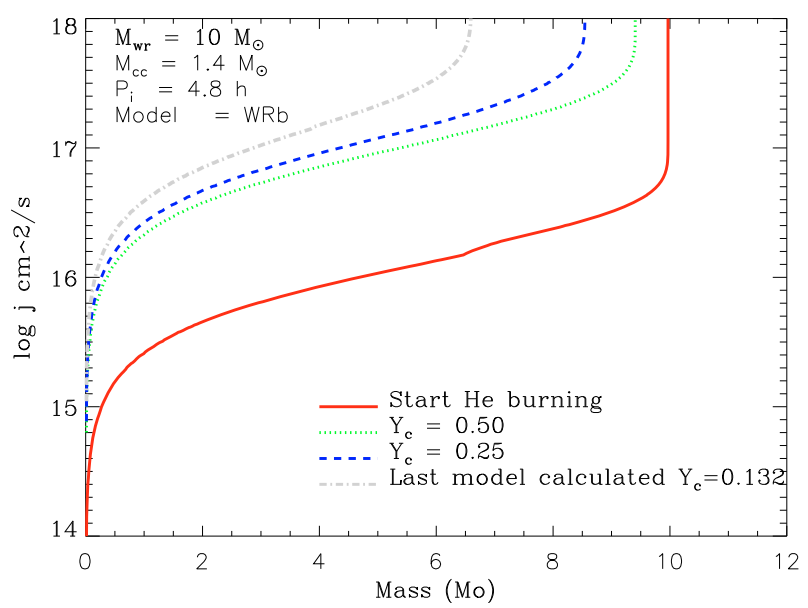

Fig. B.2. Specific angular momentum as a function of mass coordinate for Cyg X-3 model WRb (1.4 $M_{\odot}$ companion). Profiles are for the start of core helium burning (solid line), 50\% He-depletion in the core (dotted line), $25 \%$ He-depletion in the core(dashed line) and the last model calculated when RLOF started (dot-dashed line).

\section{Appendix B: Angular momentum evolution}

The detailed specific angular momentum profiles of the WR stars for each model (WRa, WRb and WRc) can be seen in Figs. B.1B.3. These all show that during core helium burning the specific angular momentum profile inside the star does not change significantly. Also clear is that the specific angular momentum of model WRa, does not increase during the evolution of the system, while for models WRb and WRc the spin-up clearly works, i.e. the specific angular momentum increases.

Figures B.4 and B.5 show the evolution of the orbital angular momentum and the degree of synchronization $\left(\omega_{\mathrm{WR}} / \omega_{\mathrm{orb}}\right)$. The angular momentum of the orbit always decreases, due to either the strong mass loss of the WR star (WRa) or tidal spin-up $(\mathrm{WRb}+\mathrm{WRc})$. The system remains synchronized throughout its evolution till RLOF starts.

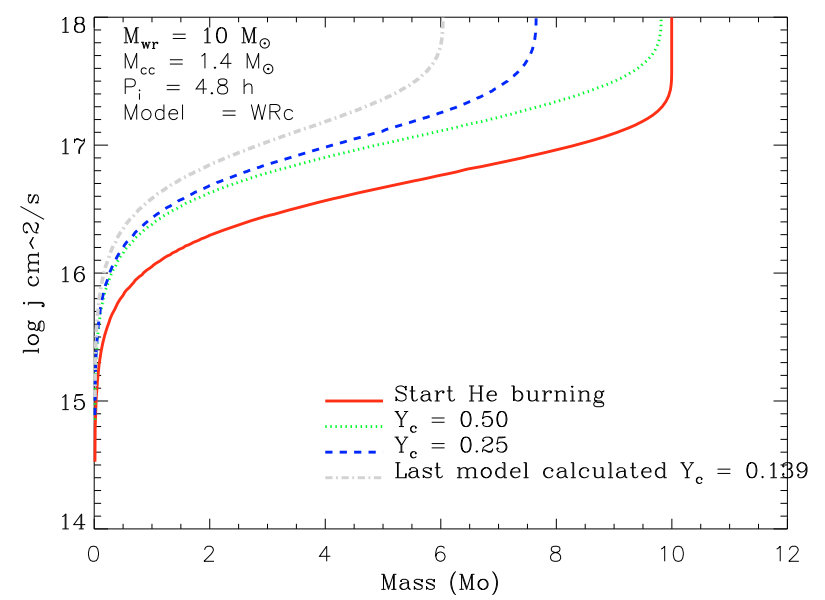

Fig. B.3. Specific angular momentum as a function of the mass coordinate for Cyg X-3 model WRc (1.4 $M_{\odot}$ companion). Profiles are given for the start of core helium burning (solid line), 50\% He-depletion in the core (dotted line), 25\% He-depletion in the core (dashed line) and the last model calculated when RLOF started (dot-dashed line).

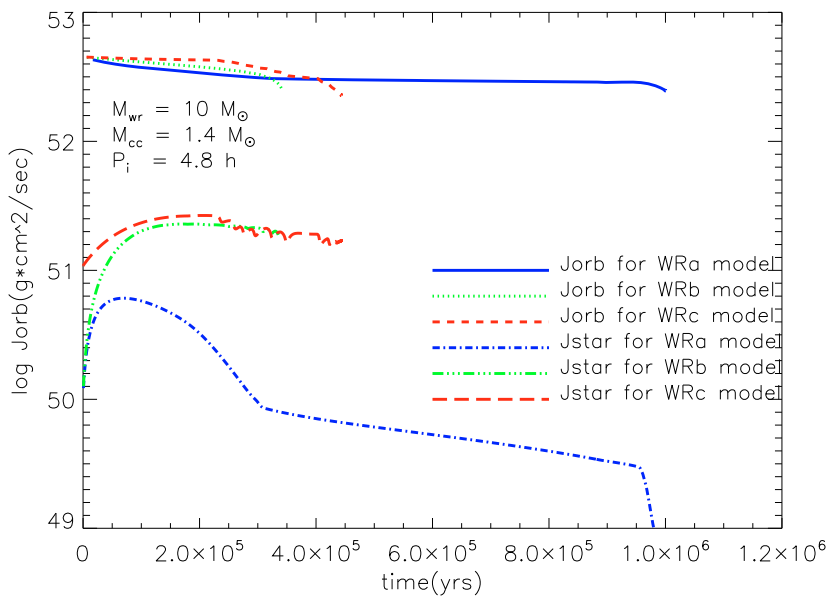

Fig. B.4. Orbital and stellar angular momentum as a function of time for our three Cyg X-3 models. The orbital angular momentum (solid lines) is plotted for model WRa (blue, solid), model WRb (green,dashed) and model WRc (red,dotted). The stellar angular momentum (dotted lines) has the same coloring for the different models.

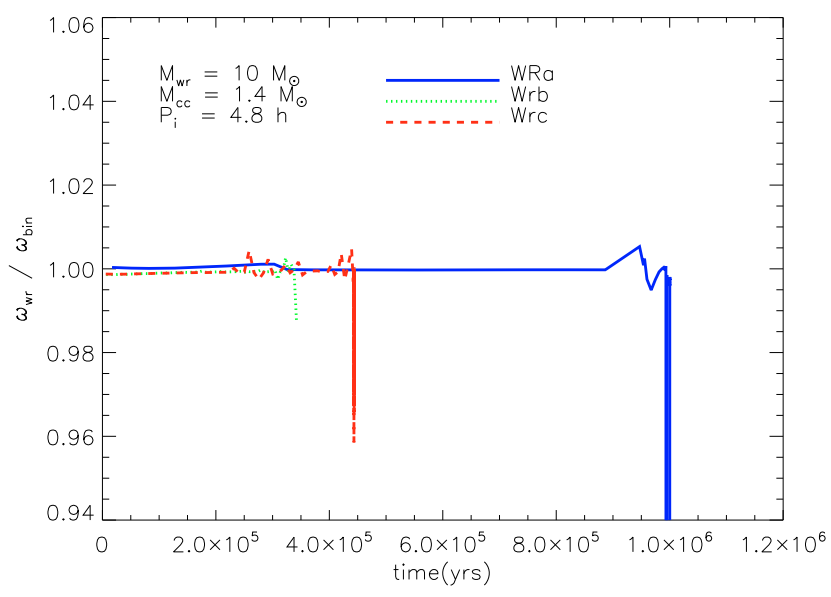

Fig. B.5. The ratio of angular velocity and orbital angular velocity $\omega_{\text {wr }} / \omega_{\text {bin }}$ for model WRa (solid blue line), model WRb (dotted green line) and model WRc (dashed red line). 


\section{References}

Belczynski, K., Kalogera, V., \& Bulik, T. 2002, ApJ, 572, 407

Cherepashchuk, A., \& Postnov, K. 2001, in Gamma-ray Bursts in the Afterglow

Era, ed. E. Costa, F. Frontera, \& J. Hjorth, 166

de Kool, M. 1990, ApJ, 358, 189

Fryer, C. L., \& Heger, A. 2005, ApJ, 623, 302

Fryer, C. L., Woosley, S. E., \& Hartmann, D. H. 1999, ApJ, 526, 152

Hamann, W.-R., Schoenberner, D., \& Heber, U. 1982, A\&A, 116, 273

Hamann, W.-R., Koesterke, L., \& Wessolowski, U. 1995, A\&A, 299, 151

Heger, A., Woosley, S. E., \& Spruit, H. C. 2005, ApJ, 626, 350

Hirschi, R., Meynet, G., \& Maeder, A. 2006, ArXiv Astrophysics e-prints

Hjorth, J., Sollerman, J., Møller, P., et al. 2003, Nature, 423, 847

Hurley, J. R., Tout, C. A., \& Pols, O. R. 2002, MNRAS, 329, 897

Kroupa, P., Tout, C. A., \& Gilmore, G. 1993, MNRAS, 262, 545

Langer, N. 1997, in Luminous Blue Variables: Massive Stars in Transition, ASP Conf. Ser., 120, 381

Langer, N. 1998, A\&A, 329, 551

Lommen, D., Yungelson, L., van den Heuvel, E., Nelemans, G., \& Portegies Zwart, S. 2005, A\&A, 443, 231

MacFadyen, A. I., \& Woosley, S. E. 1999, ApJ, 524, 262
Nugis, T., \& Lamers, H. J. G. L. M. 2000, A\&A, 360, 227

Packet, W. 1981, A\&A, 102, 17

Petrovic, J., Langer, N., \& van der Hucht, K. A. 2005, A\&A, 435, 1013

Spruit, H. C. 2002, A\&A, 381, 923

Stark, M. J., \& Saia, M. 2003, ApJ, 587, L101

Suijs, M., Langer, N., Yoon, S.-C., Poelarends, A.-J., \& Heger, A. 2008, in prep.

Thorne, K. S., \& Żytkow, A. N. 1975, ApJ, 199, L19

Toledano, O., Moreno, E., Koenigsberger, G., Detmers, R., \& Langer, N. 2007, A\&A, 461, 1057

van den Heuvel, E. P. J., \& Yoon, S.-C. 2007, Ap\&SS, 311, 177

Van Kerkwijk, M. H., Geballe, T. R., King, D. L., van der Klis, M., \& van Paradijs, J. 1996, A\&A, 314, 521

van Marle, A. J., Langer, N., \& García-Segura, G. 2005, A\&A, 444, 837

Vink, J. S., \& de Koter, A. 2005, A\&A, 442, 587

Vrancken, M., de Greve, J. P., Yungelson, L., \& Tutukov, A. 1991, A\&A, 249, 411

Webbink, R. F. 1984, ApJ, 277, 355

Wellstein, S. 2001, Ph.D. Thesis, University of Potsdam

Woosley, S. E. 1993, BAAS, 25, 894

Yoon, S.-C., \& Langer, N. 2005, A\&A, 443, 643

Yoon, S.-C., Langer, N., \& Norman, C. 2006, A\&A, 460, 199

Zahn, J.-P. 1977, A\&A, 57, 383 